\title{
Effect of COVID-19 on patients with compensated chronic liver diseases
}

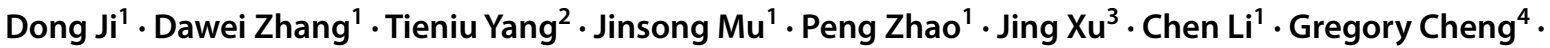 \\ Yudong Wang ${ }^{4} \cdot$ Zhu Chen $^{1} \cdot$ Enqiang Qin ${ }^{1}$ - George Lau ${ }^{1,4}$
}

Received: 19 May 2020 / Accepted: 20 May 2020 / Published online: 30 July 2020

(c) Asian Pacific Association for the Study of the Liver 2020

\begin{abstract}
Background and Aim Cytokine storm has been reported in patients with coronavirus disease 2019 (COVID-19) due to severe acute respiratory syndrome coronavirus-2 (SARS-CoV-2) infection. We examine the incidence of acute on chronic liver failure (ACLF) in COVID-19 patients with pre-existing compensated chronic liver disease (CLD).

Methods From 20 Jan 2020 to 7 Feb 2020, we studied 140 consecutive COVID-19 patients admitted to either Fuyang Second People's Hospital (FYSPH), Anhui or the Fifth Medical Center of Chinese PLA General Hospital (PLAGH) in Beijing, China. Pre-existing CLD includes those with liver cirrhosis assessed by APRI/FIB-4 score and /or ultrasound; NAFLD as identified by either ultrasound or hepatic steatosis index with significant liver fibrosis and chronic hepatitis B (CHB) or hepatitis C (CHC) infection. The diagnosis, grading of severity and clinical management of COVID-19 patients complied to the guideline and clinical protocol issued by the China National Health Commission. All patients had liver function test at least twice weekly till discharge with full recovery or death.

Results In total, 3 had liver cirrhosis, 6 patients had CHB, 13 had NAFLD with significant liver fibrosis (one also had CHB). On admission, none had liver decompensation. COVID-19 disease progression was significantly less frequent in non-CLD patients $(10 / 1188.5 \%)$ than CLD patients $(13 / 2259.1 \%, p<0.001)$. One patient with CLD had acute-on-chronic liver failure (ACLF).

Conclusion Disease progression is significantly higher in those COVID-19 patients with CLD as compared to those with no CLD. ACLF can also occur in patient with pre-existing compensated CLD who had severe COVID-19.
\end{abstract}

Keywords Acute-on-chronic liver failure · Chronic liver diseases · COVID-19

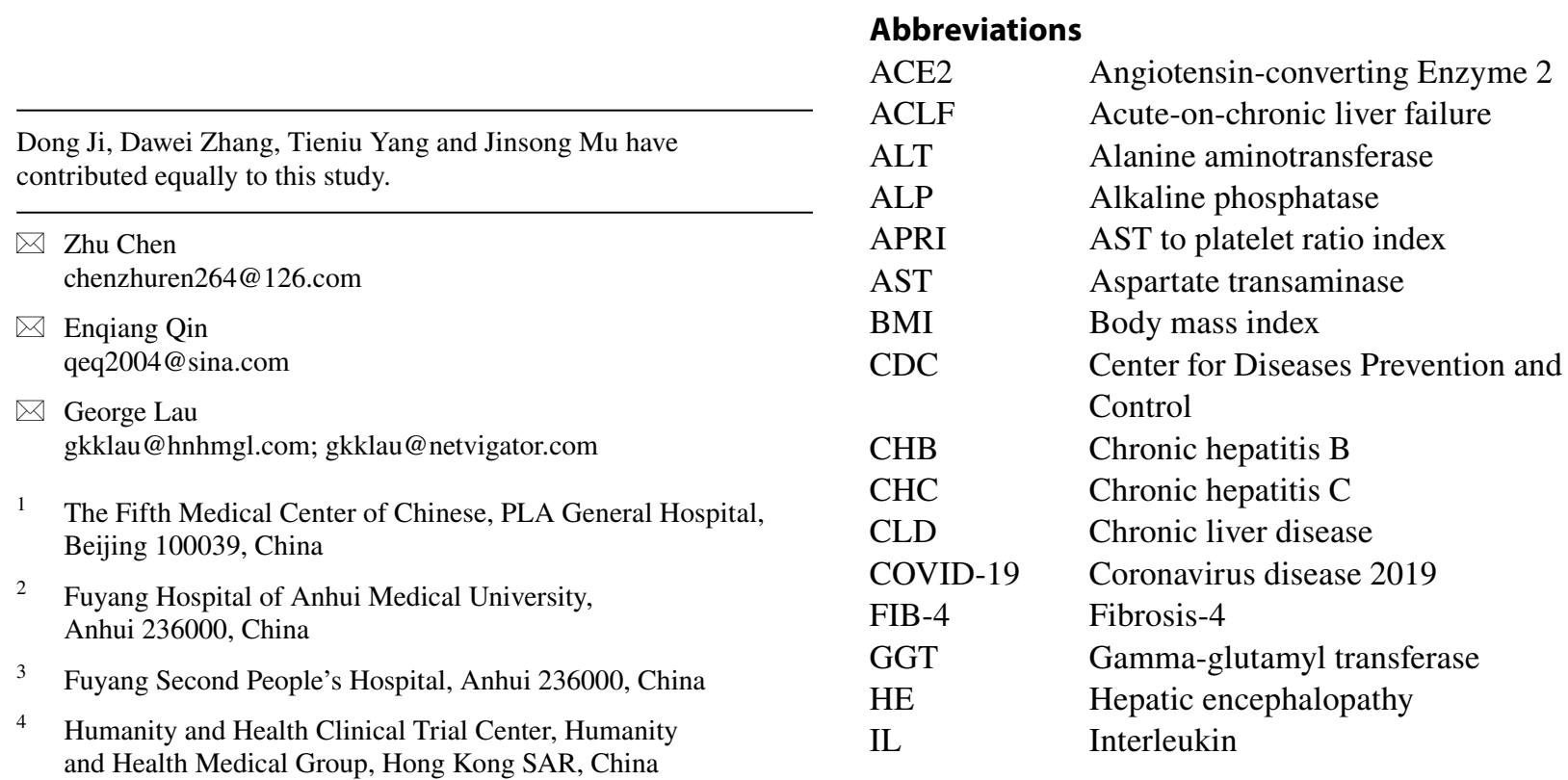


NAFLD Non-alcoholic fatty liver disease

NUCs Nucleos(t)ide analogues

RT-PCR Real-time reverse-transcriptase polymerase chain reaction

SARS-CoV-2 Severe acute respiratory syndrome coronavirus-2

TBIL Total bilirubin

\section{Introduction}

With the rapidly expanding coronavirus disease 2019 (COVID-19) pandemic, liver injury has been increasingly recognized as part of the clinical picture. Indeed, hepatitis with elevation of serum alanine transaminases (ALT) has been reported to occur in up to half of the patients infected by severe acute respiratory syndrome coronavirus 2 (SARSCoV-2) $[1,2]$. The severity and frequency of liver injury correlate to the severity of COVID-19; those with disease progression suffered from severe liver injury more frequently [3-5]. The underlying mechanism of the liver injury remains speculative. In influenza, severe hepatitis and acute hepatic decompensation in patients with underlying chronic liver disease (CLD) has been reported [6]. As no virus was found in the liver, "collateral damage" resulting from expanding $\mathrm{CD}^{+} \mathrm{T}$ cell populations during the influenza infections has been implicated for liver pathobiology [7]. In COVID-19, there are cumulating immunological evidences to suggest that inflammation serves as an important driver of disease progression. A phenomenon termed "cytokine storm" characterized by markedly elevated plasma levels of pro-inflammatory cytokines such as interleukin (IL)-2, IL-6, granulocyte-colony stimulating factor, interferon- $\gamma$ inducible protein 10 , monocyte chemoattractant protein 1 , macrophage inflammatory protein 1- $\alpha$, and tumour necrosis factor- $\alpha$, has been associated with severe COVID-19 [3-5, 8]. These findings bear resemblance to SARS caused by the SARS coronavirus, another closely related coronavirus, where cytokine storm and other dysregulated immune responses have been associated with disease pathogenesis [9]. On the other hand, direct cytopathic damage by the SARS-CoV-2 is also possible as there are entry receptors angiotensin-converting enzyme 2 (ACE2) in the liver, especially in the bile duct cells [10]. Also, learning from SARS experience, the use of antibiotics, antiviral such as lopinavir/litonavir, other traditional Chinese medicine and secondary bacterial infection, might lead to liver injury in COVID-19 patients [11]. Globally, there is an increasing prevalence of CLD with underlying advanced fibrosis or cirrhosis, due to chronic hepatitis $\mathrm{B}$ or $\mathrm{C}$ infection and recently, non-alcoholic and alcoholic fatty liver [12]. It is also unknown whether the disturbance of immunity related to COVID-19, will have an effect of hepatitis B or C infection, leading to reactivation [13, 14]. Many of these CLD patients are asymptomatic in a "compensated" state. With a reduced liver function reserve, these patients with CLD run a higher risk of decompensation with liver failure. So far, there is a lack of reports on acute-on-chronic liver failure (ACLF) in COVID-19 patients with compensated CLD. ACLF is a syndrome characterized by acute decompensation of CLD associated with organ failures and high short-term mortality. Nevertheless, there are great heterogeneity in the definition of 'acute', 'chronic liver' and 'failure' by different working parties on ACLF [15]. In 2009, the Asian Pacific Association for the Study of the Liver (APASL) provided the first consensus on ACLF, defined as "an acute hepatic insult manifesting as jaundice and coagulopathy, complicated within 4 weeks by ascites and/or encephalopathy" [16]. The 2014 definition was further expanded to include 'high 28-day mortality' [17]. The main difference with all other definitions is that hepatic insults are only taken in consideration if they lead to liver failure (jaundice and HE). In this study, we report the incidence of liver injury and ACLF in patients with CLD compared with those without CLD, in accordance to APASL guidelines in Chinese with COVID-19.

\section{Methods}

\section{Patients studied}

Previously, we had studied patients presented to either Fuyang Second People's Hospital (FYSPH) in Anhui or the Fifth Medical Center of Chinese PLA General Hospital (PLAGH) in Beijing, China for the impact of non-alcoholic fatty liver diseases (NAFLD) in patients with COVID-19 [18]. From this cohort of patients, we reviewed their medical records for previous ultrasound studies, chronic hepatitis B infections and performed AST to platelet ratio index (APRI), Fibrosis-4 (FIB-4), hepatic steatosis index (HSI) and BARD-Score to identify subjects with chronic liver disease (CLD). All COVID-19 patients without CLD admitted to these two hospitals during the same period as the COVID-19 patients with CLD were used for comparisons. These COVID-19 patients were follow-up till discharged with recovery or death (Fig. 1). The diagnosis and clinical management of COVID-19 patients were in accord to the practice guidelines "Chinese Clinical Guidance for COVID-19 Pneumonia Diagnosis and Treatment" issued by The Chinese National Health Commission, China. Briefly, the presence of SARS-CoV-2 in respiratory specimens was confirmed using real-time reverse-transcriptase polymerase chain reaction (RT-PCR) assay by local municipal Center for Diseases Prevention and Control (CDC). All patients received treatment with lopinavir/ritonavir. For those with severe symptoms, methylprednisolone (1-2 mg per $\mathrm{kg}$ weight per day) and empirical antibiotics (moxifloxacin or 


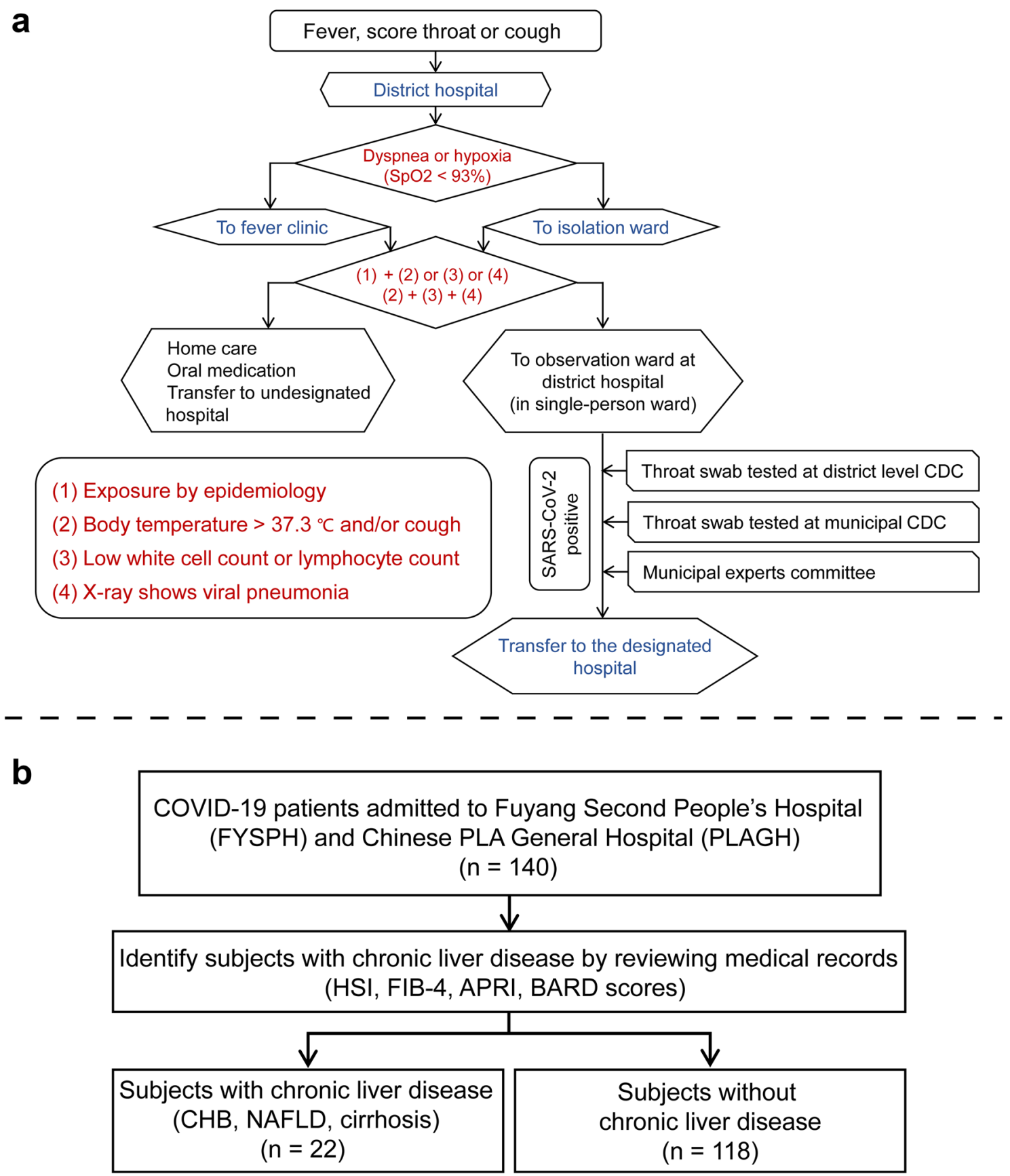

Fig. 1 a. Flow chart for management of COVID-2019 in fever clinics outside Wuhan. b Consort Diagram of study population

piperacillin/tazobactam or meropenem) would be administered according to patients' clinical characteristics and physicians' decisions. For those with respiratory failure, shock, or multi-organ failures, life support measures including ECMO were administered in an intensive care unit (ICU). Progression of illness was defined as development of at least one of the followings: respiratory rate $\geq 30$ breaths $/ \mathrm{min}$, resting oxygen saturation $\leq 93 \%$ and $\mathrm{PaO} 2 / \mathrm{FiO} 2 \leq 300 \mathrm{mmHg}$ or worsening of lung CT findings, during the hospitalization period. The criteria of hospital discharge included all the following conditions: body temperatures remain normal over 3 days, significant improvement of respiratory symptoms, resolution of pulmonary imaging of inflammation, and repeated testing for SARS-CoV-2 confirm viral clearance at least one day apart. All CHB patients who were not on anti-HBV nucleos(t)ide analogues (NUCs), will be initiated NUCs if they received immunosuppressive therapy. Viral shedding period was defined as the time to undetectable 
SARS-CoV-2 by RT-PCR from symptom onset [19]. The study was approved by the Ethics Committees of FYSPH (20200303006) and the Fifth Medical Center of PLAGH (2020005D). Written informed consent was waived in view of the designated hospital for new emerging infectious diseases status of the two centers.

\section{Data collection}

All the patients' information, such as epidemiological, demographic, clinical, laboratory, treatment and outcome data, were collected from medical records. All patients had liver biochemistries including alanine aminotransferase (ALT), aspartate transaminase (AST), total bilirubin (TBIL), alkaline phosphatase (ALP) and gamma-glutamyl transferase (GGT) on admission and then at least twice weekly till last follow-up. Comorbidity was defined as having at least one of the followings: hypertension, diabetes, coronary heart disease and chronic lung disease within 12 months of admission. Nonalcoholic fatty liver disease (NAFLD) was identified as hepatic steatosis index [HSI $=8 \times(\mathrm{ALT} /$ AST) + BMI ( +2 if type 2 diabetes yes, +2 if female)] more than 36 points and/or by abdominal ultrasound examination [20]. Chronic hepatitis B (CHB) was defined as HBsAg positive for at least 6 months. Chronic liver disease was defined as FIB- $4>3.25$ or APRI $>1.5$ or CHB or subjects with HSI $>36+$ BARD score 2-4 [21]. ACLF was defined by APASL criteria: jaundice (serum bilirubin $>85$ umol/L) and/or coagulopathy (international normalized ratio $>1.5$ ) complicated within 4 weeks by ascites or encephalopathy [17]. All the data in source documents were confirmed independently by at least two researchers.

\section{Statistical analysis}

Continuous variables were expressed as mean \pm SD and compared using the unpaired, two-tailed student's $t$ test (for normal distribution data) or median [interquartile range (IQR)] and compared with Mann-Whitney test (for skewed distribution data). Categorical variables were presented as numbers (percentage) and compared by the chi-square test or Fisher's exact test. A $p$ value $<0.05$ was considered as significant for all statistical tests. The statistical analyses were performed using R software, version 3.6.1 (R Foundation for Statistical Computing, Vienna, Austria; https:// www.r-project.org/).

\section{Results}

\section{Clinical characteristics of enrolled patients}

Altogether, 140 patients with COVID-19 presented to the 2 hospitals from 20 Jan 2020 to 7 Feb 2020 were enrolled
(Fig. 1b). The median age (IQR) was 44.1 (33.3-52.3) years and 82 out of 140 patients $(58.6 \%)$ were male. The median BMI (IQR) was $23.9(22.0-26.0) \mathrm{kg} / \mathrm{m}^{2}, 32(22.9 \%)$ had underlying comorbidities, 54 (38.6\%) had NAFLD, $7(5.0 \%)$ had positive HBsAg. The median in-hospital days were 18.0 (13.0-26.0) days. Of the 140 COVID 19 patients, 118 (84.3\%) had no CLD and 22 (15.7\%) had CLD. Among those with CLD, 3 had cirrhosis with FIB-4>3.25 \& APRI $>1.5,6$ had CHB and 13 NAFLD had significant fibrosis (HIS $>36+$ BARD scores 2-4; one also had CHB). For the seven patients with $\mathrm{CHB}$, two were already on entecavir $0.5 \mathrm{mg}$ daily before COVID-19 diagnosis (HBV DNA copies $1.7 \times 10^{3}$ and $3.6 \times 10^{5} \mathrm{IU} / \mathrm{mL}$ ) and the other three were started on entecavir $0.5 \mathrm{mg}$ daily after admission (HBV DNA copies all $\left.<1.5 \times 10^{3} \mathrm{IU} / \mathrm{mL}\right)$. Ten $(77 \%)$ NAFLD patients had diabetes as well. Compared to those without CLD, those with CLD were older, with a higher BMI and more underlying comorbidities. Those with CLD also had higher proportion of patients with disease progression (Table 1).

\section{Incidence of liver injury and dynamic changes of cytokines}

During the clinical course of COVID-19, liver injury in terms of elevated serum ALT or ALP with GGT was the same among those with CLD as compared to those without CLD. The injury pattern was mainly hepatocellular with elevated ALP noted in less than four percent of subjects in each group. The mean of ALT, AST, TBIL, ALP, IL-6 and ferritin on admission, peak after admission, and discharge were shown in Fig. 2a. IL-6 levels were significantly higher in CLD patients at all three time points $(42.5 \pm 11.9 \mathrm{pg} /$ $\mathrm{mL}$ vs. $16.2 \pm 11.3 \mathrm{pg} / \mathrm{mL}, p<0.001,68.9 \pm 46.9$ vs. $9.8 \pm 6.1 \mathrm{pg} / \mathrm{mL}, p=0.001$ and $48.4 \pm 44.8$ vs. $4.0 \pm 2.7 \mathrm{pg} /$ $\mathrm{mL}, p=0.006$, respectively). No significant differences observed with ALT, AST, ALP and TBL between the two groups. Serum ferritin levels were also higher in CLD group at admission $(442.5 \pm 131.0 \mathrm{ug} / \mathrm{L}$ vs. $285.4 \pm 285.0 \mathrm{ug} / \mathrm{L}$, $p=0.034)$ and at peak values after admission $666.4 \pm 364.7$ ug/L vs. $410.9 \pm 391.6 \mathrm{ug} / \mathrm{L}, p=0.049$ ) (Fig. 2a). Among CLD patients with or without CHB, there were no difference in ALT, AST, ALP and TBL, serum ferritin and IL-6 on admission, peak and last follow-up (Fig. 2b).

\section{COVID-19 patients who had ACLF}

A 79-year-old female with pre-existing NAFLD with fibrosis and no previous history of diabetes. She had a 4-day history of fever, cough with expectoration. SARS-CoV-2 infection was confirmed by real-time PCR assay from a throat swab sample. On admission, liver enzymes were normal. Chest $\mathrm{X}$-ray showed multiple patchy ground glass opacities in 
Table 1 Comparison of COVID-19 Patients with and without CLD

\begin{tabular}{llllr}
\hline & Overall $(n=140)$ & $\begin{array}{l}\text { Non-CLD group } \\
(n=118,84.3 \%)\end{array}$ & $\begin{array}{l}\text { CLD group } \\
(n=22,15.7 \%)\end{array}$ & $p$ value \\
\hline Male sex & $82(58.6)$ & $68(57.6)$ & $14(63.6)$ & 0.772 \\
Age (years) & $43.6 \pm 14.1$ & $41.9 \pm 14.0$ & $53.0 \pm 14.5$ & 0.001 \\
BMI $\left(\mathrm{Kg} / \mathrm{m}^{2}\right)$ & $24.0 \pm 2.8$ & $23.5 \pm 2.6$ & $26.6 \pm 2.1$ & $<0.001$ \\
Smoker & $13(9.3)$ & $11(9.3)$ & $2(9.1)$ & 1.000 \\
Drinker & $5(3.6)$ & $5(4.2)$ & $0(0.0)$ & 1.000 \\
Comorbidities & $32(22.9)$ & $19(16.1)$ & $13(59.1)$ & $<0.001$ \\
HBsAg positive & $7(5.0)$ & $0(0.0)$ & $7(31.8)$ & $<0.001$ \\
NAFLD & $54(38.6)$ & $35(29.7)$ & $19(86.4)$ & $<0.001$ \\
Admission & & & & \\
Elevated ALT $(n, \%)$ & $74(52.9)$ & $59(50.0)$ & $15(68.2)$ & 0.182 \\
Elevated AST $(n, \%)$ & $25(17.9)$ & $19(16.1)$ & $6(27.3)$ & 0.341 \\
Elevated ALP $(n, \%)$ & $4(2.9)$ & $4(3.4)$ & $0(0.0)$ & 1.000 \\
Elevated TBIL $(n, \%)$ & $9(6.4)$ & $7(5.9)$ & $2(9.1)$ & 0.632 \\
APRI & $0.48 \pm 0.35$ & $0.46 \pm 0.34$ & $0.59 \pm 0.38$ & 0.108 \\
FIB-4 & $1.50 \pm 0.84$ & $1.42 \pm 0.80$ & $1.91 \pm 0.92$ & 0.012 \\
BARD score & $1.48 \pm 1.10$ & $1.35 \pm 1.10$ & $2.18 \pm 1.40$ & 0.002 \\
In-hospital days & $18.0(13.0-26.0)$ & $16.5(12.3-25.5)$ & $19.5(15.5-26.0)$ & 0.116 \\
COVID-19 progression & $23(16.4)$ & $10(8.5)$ & $13(59.1)$ & $<0.001$ \\
Death/discharge & $1 / 139$ & $0 / 118$ & $1 / 21^{\mathrm{b}}$ & 0.157 \\
\hline
\end{tabular}

Continuous variables were expressed as mean \pm SD and compared using the unpaired, two-tailed Student's $t$ test (for normal distribution data) or median [interquartile range (IQR)] and compared with Mann-Whitney test (for skewed distribution data). Categorical variables were presented as numbers (percentage) and compared by the chi-square test or Fisher's exact test

ALT alanine aminotransferase; AST, aspartate transaminase; ALP, alkaline phosphatase; GGT, glutamyl transferase; TBIL, total bilirubin

${ }^{a}$ Comorbidity included hypertension, diabetes, cardiovascular disease and chronic lung disease

${ }^{\mathrm{b}}$ One subject with cirrhosis passed away both lungs (Fig. 3). Oxygen therapy, methylprednisolone, human $\gamma$-Immune goblins, moxifloxacin and other symptomatic treatments were given according to the practice guidelines "Chinese Clinical Guidance for COVID-19 Pneumonia Diagnosis and Treatment" issued by The Chinese National Health Commission, China. Her serum IL-6 increased rapidly to $159.1 \mathrm{pg} / \mathrm{mL}$ on day 4 and ferritin level increased to $>780 \mathrm{ug} / \mathrm{L}$. On day 6 , she had increasing dyspnea and serum creatinine increased from 64.4 to $126 \mathrm{mmol} / \mathrm{L}$. Chest $\mathrm{X}$-ray showed progression of multiple patchy shadows in both lungs. The patient was transferred to ICU for invasive mechanical ventilation (IMV) and continuous renal replacement therapy (CRRT). Her bilirubin rose to $132 \mathrm{mmol} / \mathrm{L}$ fulfilling the APASL criteria of ACLF on day 9. On Day 12 , her respiratory symptoms and liver functions improved. She became afebrile and CT showed significant resolution of the multiple patchy shadows. IMV was discontinued. However, her serum IL-6 increased markedly to $200.7 \mathrm{pg} / \mathrm{mL}$ on day 12 and ferritin continued to increase to $>1444 \mathrm{ug} / \mathrm{L}$ on day 14. She developed fever with temperature $38.2{ }^{\circ} \mathrm{C}$, melena and hypotension on day 14 . She was treated empirically as septic shock with multiple antibiotics (tigecycline, vancomycin, capofungin, imipenum and cilastatin sodium). The liver function deteriorated again on day 16 followed by development of ascites. The patient succumbed on day 22 with multi-organ failure. SARS-CoV-2 sequences were no longer detectable in patient's specimens by RT-PCR from day 20 onwards (Fig. 3). The bilirubin, INR and IL6 levels at the time of death on Day 22 were 164.9 umol/L, 4.3 and $>500 \mathrm{pg} / \mathrm{ml}$, respectively (Fig. 3).

\section{Discussion}

To date, there has been no report on the incidence of liver injury and ACLF in CLD patients with COVID-19. In our present report, we evaluate the pattern of liver injury and the occurrence of ACLF in those COVID-19 patients with pre-existing asymptomatic CLD. There were no significant differences in terms of hepatocellular or ductular injury, as reflected by the incidence and pattern of elevation of serum ALT or ALP, respectively among patients with or without CLD. However, COVID-19 patients with CLD was found to have a higher level of pro-inflammatory cytokine, such 

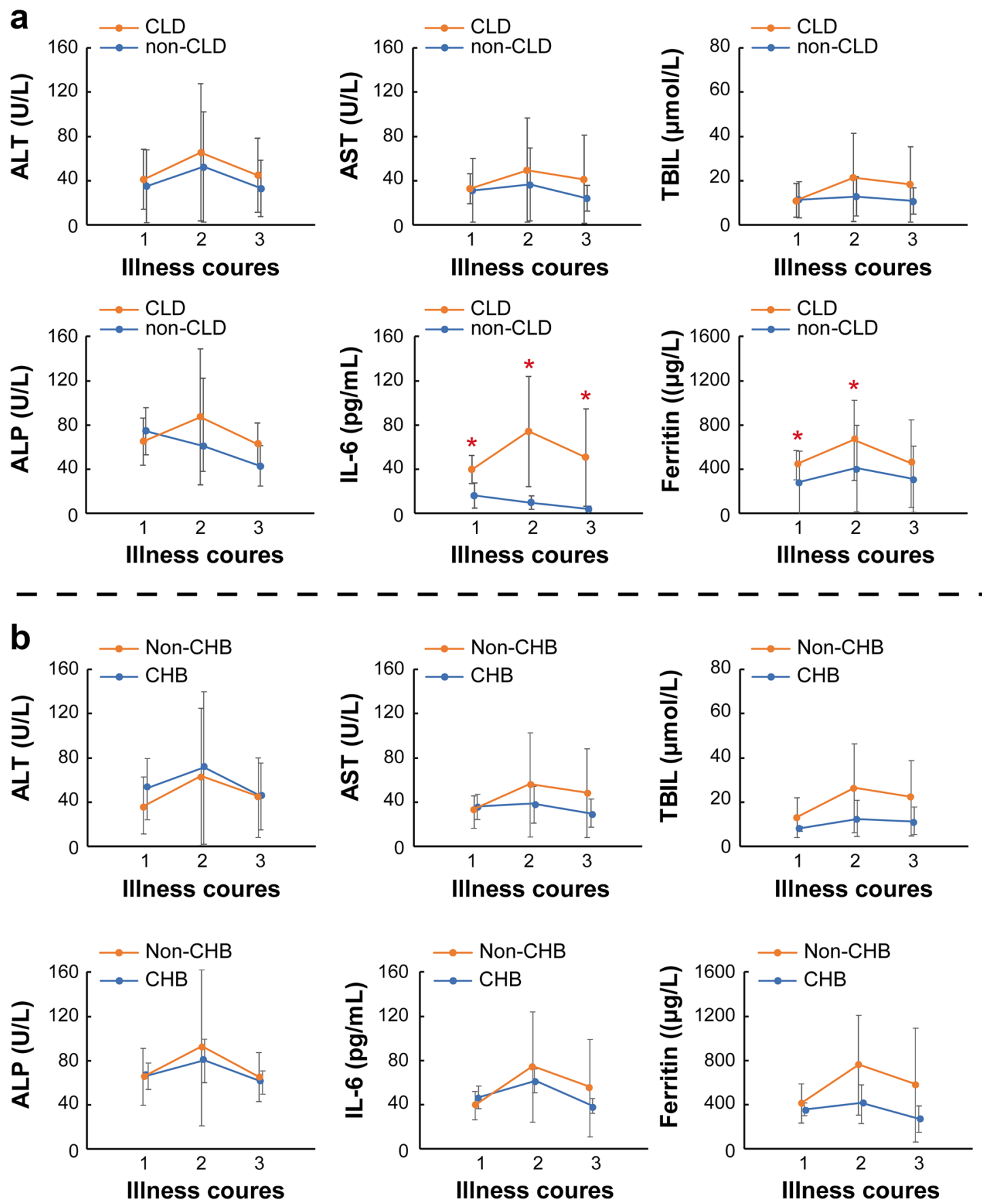

Fig. 2 Profiles of liver enzymes and inflammatory markers in COVID-19 patients (a) with and without chronic liver disease and (b) with or without $\mathrm{CHB}$, at three time points (1: at admission, 2: peak values after admission and 3: at last follow-up)

as IL-6 and ferritin. This is in keeping with the previous observation that elevation of serum level of TNF-alpha and IL-6 was significantly higher in cirrhotic as compared to those without liver disease on exposure to sepsis [22]. Most of the clinical reports in COVID-19 patients, had shown an elevation of proinflammatory marker in severe COVID19. ${ }^{1-5,8,9}$ Indeed, "cytokine storm" has been consistently reported to occur in severe or fatal COVID-19, as in SARS 
[23]. In our study, CLD also had a significantly higher risk of disease progression. This further supports the inclusion of CLD as a comorbidity risk factor for disease progression in COVID-19 [18, 24].

There was one patient with CLD who developed ACLF and subsequently succumb after clearance of SARSCoV-2. The underlying mechanism is not clear. In an autopsy performed on a 50-year-old man with severe pneumonia caused by SARS-CoV-2, the liver tissue showed only moderate microvesicular steatosis and mild lobular activity, but there was no conclusive evidence to support SARS-CoV-2 direct cytopathic effect or immune mediated injury [25]. There was also no mention of liver dysfunction in the report. However, the authors found that even though peripheral $\mathrm{CD}^{+}$and $\mathrm{CD}^{+} \mathrm{T}$ cells were substantially reduced and they were in a hyperactivated status, as evidenced by the high proportions of HLA-DR (CD4 $3.47 \%$ ) and CD38 (CD8 39.4\%) double-positive fractions. Also, there was an increased concentration of highly proinflammatory Th17 in the $\mathrm{CD}^{+}{ }^{+} \mathrm{T}$ cells in the diseased patient favoring severe immune injury. In another report of post-mortem needle core biopsies of liver in four patients who died of COVID-19 pneumonia, the liver sections only showed mild sinusoidal dilatation, a common non-specific change in terminally ill hospitalized patients [26]. Focal macrovesicular steatosis, and dense atypical small lymphocytes in portal tracts were seen in a patient with medical history of chronic lymphocytic leukemia. In general, there was no significant lymphocytic infiltration of the portal tracts. No obvious fatty changes were seen in three of the four patients. Liver tissue from one patient contained regenerative nodules and thick fibrous bands, consistent with history of cirrhosis. One patient also showed patchy hepatic necrosis in the periportal and centrilobular areas. Again, there was no mentioned of ACLF in these four cases. Our patient had NAFLD with fibrosis. She developed multiple organ failures and eventually succumbed despite clearance of SARS-CoV-2. She had normal liver enzymes on admission, then developed jaundice and coagulopathy meeting APASL criteria of ACLF. With the recovery from respiratory distress and clearing of Chest $\mathrm{X}$-ray abnormalities, her liver function and coagulopathy transiently improved. However, subsequently her disease followed a rapid progressive course. The underlying mechanism of liver damage was not clear, but both episodes were preceded by upregulation of inflammatory markers, IL-6 and ferritin in the serum in keeping with previous reports of high levels of TNF- $\alpha$ and IL-6 in ACLF patients with systemic inflammation [27]. Our study also showed that serum IL6 levels were significantly higher in COVID-19 subjects with CLD compared to those without CLD. Direct cytopathic liver damage by the SARSCoV-2 was possible, but SARS-CoV-2 sequences were no longer detectable in nasal swab specimens by RT-PCR from day 20 to day 22 , the time period of the most rapid rise in liver enzymes and marked worsening of coagulopathy, At the same time, the serum IL6 and serum ferritin levels increased rapidly reaching highest levels on day of death, therefore favouring immune mediated attacks rather than direct cytopathic effects. Also, there was no conclusive evidence of direct cytopathic damage in any of the published autopsy cases [25, 26]. Hypoxic liver injury (hypoxic hepatitis) was another possible cause of deterioration of liver functions in the terminal phase of our patient. Hypoxic liver injury was reported in up to $2.5 \%$ of ICU patients, characterised by rapid transient increase of transaminases to greater than 20 times upper limit of normal and centrolobular necrosis [28]. Although hypoxia induced injury and other mechanisms, such as drug, traditional Chinese medication, sepsis, may contribute to the development of liver failure in our subject, the temporal relationship of elevation of inflammatory markers with clinical deterioration suggested that excessive systemic inflammation is likely to be the driver for the development of ACLF in COVID-19.

Our observation showed that ACLF could occur in COVID-19 patients with CLD. Further studies should be conducted so that more data can be collected to allow us to understand the full picture of COVID-19 on patients with CLD.

The shortcoming of the present study is that the presence of cirrhosis and advanced liver fibrosis was not detected by invasive liver biopsy. Instead, previously validated non-invasive markers, such as APRI score and 
Day 0

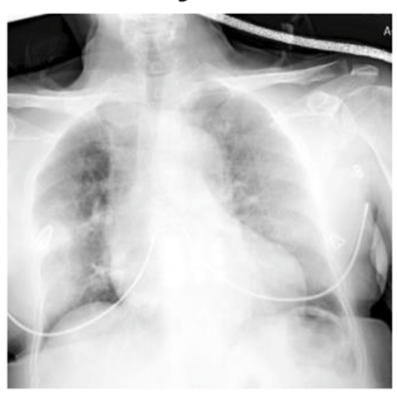

Day 14

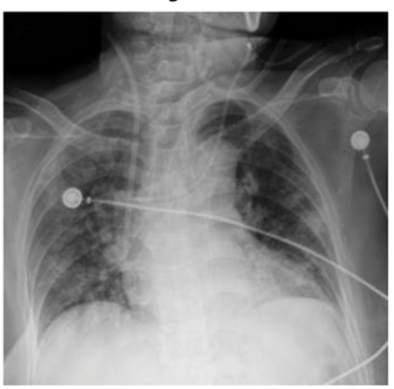

Day 3

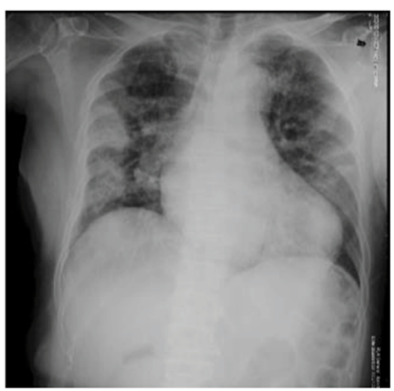

Day 15

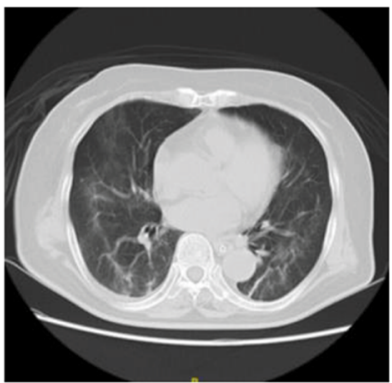

Day 4

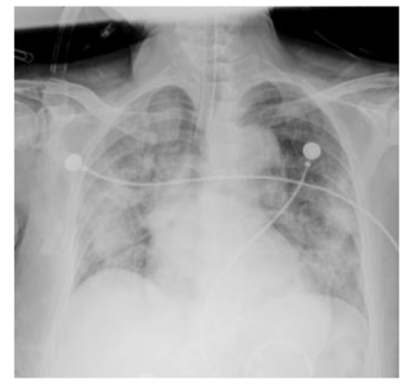

Day 21

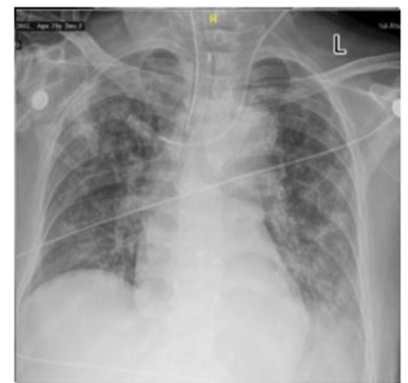

Day 6

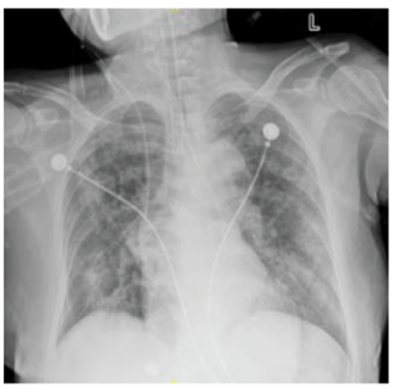

Day 22
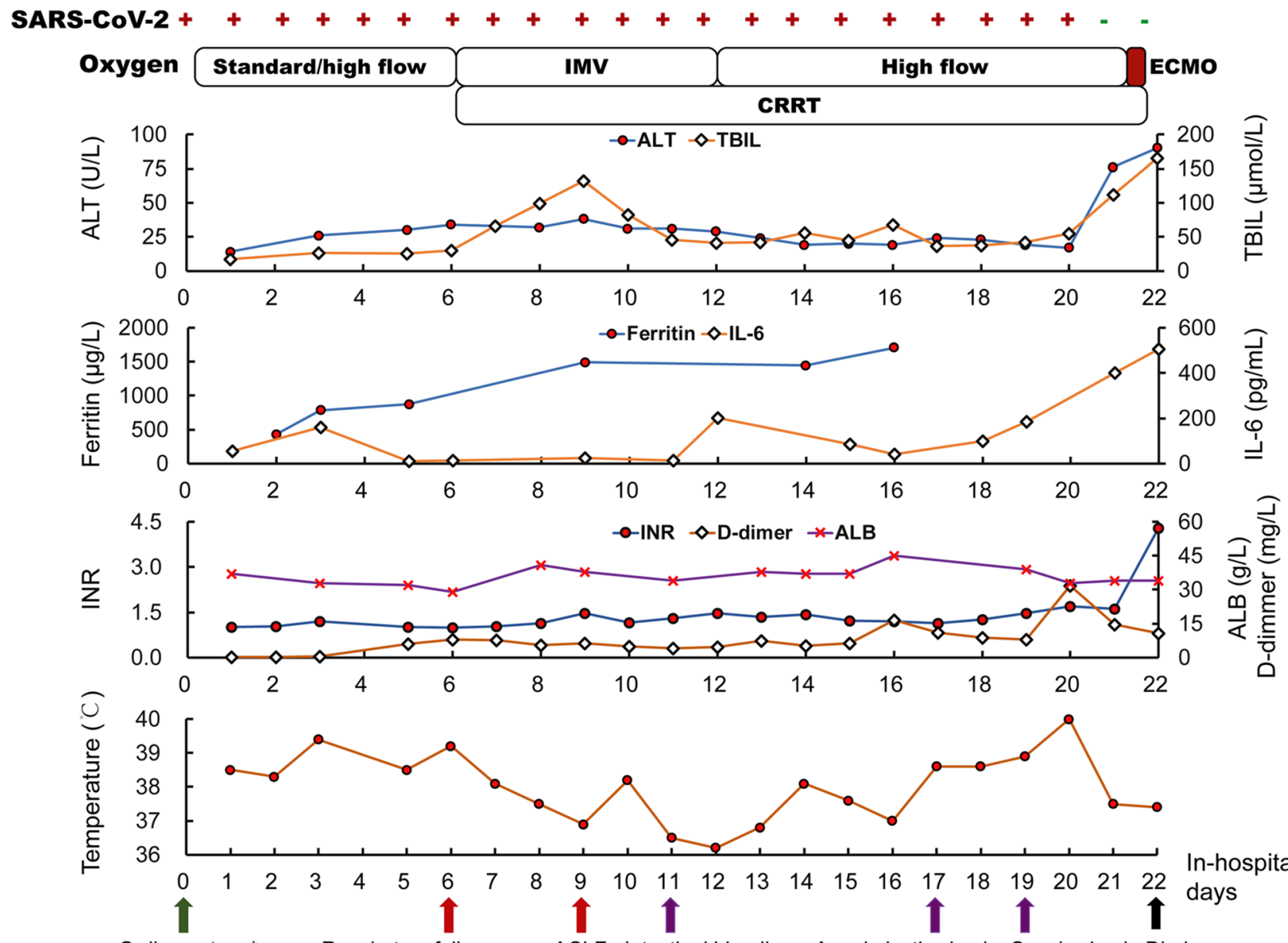

In-hospital

Ordinary type* Respiratory failure

ACLF Intestinal bleeding Anaphylactic shock Sepsis shock Died Acute kidney injury Hemorrhagic shock 
4Fig. 3 A COVID 19 patients with ACLF (IMV-invasive mechanical ventilation; CRRT-continuous renal replacement therapy; ECMOextracorporeal membrane oxygenation)

FIB-4 was used to assess the presence of underlying liver cirrhosis [29]. Further studies should be conducted so that more data can be collected to allow us to understand the full picture of COVID-19 on patients with CLD.

Acknowledgements We acknowledge all patients and health-care workers involved in the diagnosis and treatment of patients with COVID-19 in our hospitals.

Author contributors DJ, DZ, TY, JM, PZ, JX, CL, ZC and EQ treated the patients. DJ, DZ, JX, and TY collected the epidemiological and clinical data. DJ, GC, YW and GL processed statistical data and drafted the manuscript. ZC, EQ and GL had the idea for and designed the study. ZC, EQ and GL revised the final manuscript and are responsible for summarizing all the data.

Funding This work is funded by the Capital Characteristic Clinic Project of Beijing Municipal Science and Technology Commission (Z181100001718034) and Key Project of Jumei Special Fund for Hepatobiliary Disease Prevention and Treatment (2018JM12603003).

\section{Compliance with ethical standards}

Conflict of interest Dong Ji, Dawei Zhang, Tieniu Yang, Jinsong Mu, Peng Zhao, Jing Xu, Chen Li, Gregory Cheng, Yudong Wang, Zhu Chen, Enqiang Qin, George Lau declare that they have no conflict of interest.

Ethical approval This study was conducted according to the 1975 Declaration of Helsinki and approved by the ethics committee at both hospitals.

Informed consent Written informed consent was waived in view of the designated hospital for new emerging infectious diseases.

\section{References}

1. Xu L, Liu J, Lu M, Yang D, Zheng X. Liver injury during highly pathogenic human coronavirus infections. Liver Int 2020;40:998-1004.

2. Boettler T, Newsome PN, Mondelli MU, Maticic M, Cordero E, Cornberg $\mathrm{M}$, et al. Care of patients with liver disease during the COVID-19 pandemic: EASL-ESCMID position paper. JHEP Rep 2020;2:100113.

3. Zhou F, Yu T, Du R, Fan G, Liu Y, Liu Z, et al. Clinical course and risk factors for mortality of adult inpatients with COVID19 in Wuhan, China: a retrospective cohort study. Lancet 2020;395:1054-1062.

4. Wang D, Hu B, Hu C, Zhu F, Liu X, Zhang J, et al. Clinical characteristics of 138 hospitalized patients with 2019 novel Coronavirus-infected pneumonia in Wuhan, China. JAMA 2019. https:// doi.org/10.1001/jama.2020.1585.

5. Xu XW, Wu XX, Jiang XG, Xu KJ, Ying LJ, Ma CL, et al. Clinical findings in a group of patients infected with the 2019 novel coronavirus (SARS-Cov-2) outside of Wuhan, China: retrospective case series. BMJ 2020;368:m606.
6. Schütte A, Ciesek S, Wedemeyer H, Lange CM. Influenza virus infection as precipitating event of acute-on-chronic liver failure. J Hepatol 2019;70:797-799.

7. Polakos NK, Cornejo JC, Murray DA, Wright KO, Treanor JJ, Crispe IN, et al. Kupffer cell-dependent hepatitis occurs during influenza infection. Am J Pathol 2006;168:1169-1178.

8. Cao X. COVID-19: immunopathology and its implications for therapy. Nat Rev Immunol 2020;20:269-270.

9. Perlman S, Dandekar AA. Immunopathogenesis of coronavirus infections: implications for SARS. Nat Rev Immunol 2005;5:917-927.

10. Chai X, Hu L, Zhang Y, Han W, Lu Z, Ke A, et al. Specific ACE2 expression in cholangiocytes may cause liver damage after 2019-nCoV infection. BioRxiv 2020. https://doi. org/10.1101/2020.02.03.931766.

11. Zhang C, Shi L, Wang FS. Liver injury in COVID-19: management and challenges. Lancet Gastroenterol Hepatol 2020;5:428-430.

12. Asrani SK, Devarbhavi H, Eaton J, Kamath PS. Burden of liver diseases in the world. J Hepatol 2019;70:151-171.

13. Lau G. Hepatitis B reactivation after chemotherapy: two decades of clinical research. Hepatol Int 2008;2:152-162.

14. Wang C, Ji D, Chen J, Shao Q, Li B, Liu J, et al. Hepatitis due to reactivation of hepatitis $\mathrm{B}$ virus in endemic areas among patients with hepatitis $\mathrm{C}$ treated with direct-acting antiviral agents. Clin Gastroenterol Hepatol 2017; 15:132-136.

15. Hernaez R, Solà E, Moreau R, Ginès P. Acute-on-chronic liver failure: an update. Gut 2017. https://doi.org/10.1136/gutjnl-2016312670.

16. Sarin SK, Kumar A, Almeida JA, Chawla YK, Fan ST, Garg H, et al. Acute-on-chronic liver failure: consensus recommendations of the Asian Pacific Association for the study of the liver (APASL). Hepatol Int 2009;3:269-282.

17. Sarin SK, Kedarisetty CK, Abbas Z, Amarapurkar D, Bihari C, Chan AC, et al. Acute-on-chronic liver failure: consensus recommendations of the Asian Pacific Association for the Study of the Liver (APASL) 2014. Hepatol Int 2014;8:453-471.

18. Ji D, Qin E, Xu J, Zhang D, Cheng G, Wang Y, et al. Implication of non-alcoholic fatty liver diseases (NAFLD) in patients with COVID-19: a preliminary analysis. J Hepatol 2020. https://doi. org/10.1016/j.jhep.2020.03.044.

19. National Health Commission (NHC) of the PRC and National Administration of Traditional Chinese Medicine of the PRC. Guidance for Corona Virus Disease 2019: Prevention, Control, Diagnosis and Management. Bejing: Peoples Medical Publishing House; 2019.

20. Lee JH, Kim D, Kim HJ, Lee CH, Yang JI, Kim W, et al. Hepatic steatosis index: a simple screening tool reflecting nonalcoholic fatty liver disease. Dig Liver Dis 2010;42:503-508.

21. Patel K, Sebastiani G. Limitations of non-invasive tests for assessment of liver fibrosis. JHEP Rep 2020;2:100067.

22. Byl B, Roucloux I, Crusiaux A, Dupont E, Devière J. Tumor necrosis factor alpha and interleukin 6 plasma levels in infected cirrhotic patients. Gastroenterology 1993;104:1492-1497.

23. Huang KJ, Su IJ, Theron M, Wu YC, Lai SK, Liu CC, et al. An interferon-gamma-related cytokine storm in SARS patients. J Med Virol 2005;75:185-194.

24. Ji D, Zhang D, Xu J, Chen Z, Yang T, Zhao P, et al. Prediction for progression risk in patients with COVID-19 pneumonia: the CALL score. Clin Infect Dis 2020. https://doi.org/10.1093/cid/ ciaa414.

25. Xu Z, Shi L, Wang Y, Zhang J, Huang L, Zhang C, et al. Pathological findings of COVID-19 associated with acute respiratory distress syndrome. Lancet Respir Med 2020;8:420-422.

26. Tian S, Xiong Y, Liu H, Niu L, Guo J, Liao M, et al. Pathological study of the 2019 novel coronavirus disease (COVID-19) 
through post-mortem core biopsies. Mod Pathol 2020. https://doi. org/10.1038/s41379-020-0536-x.

27. Albillos A, Lario M, Álvarez-Mon M. Cirrhosis-associated immune dysfunction: distinctive features and clinical relevance. J Hepatol 2014;61:1385-1396.

28. Tapper EB, Sengupta N, Bonder A. The incidence and outcomes of ischemic hepatitis: a systematic review with meta-analysis. Am J Med 2015;128:1314-1321.
29. Vilar-Gomez E, Chalasani N. Non-invasive assessment of nonalcoholic fatty liver disease: clinical prediction rules and bloodbased biomarkers. J Hepatol 2018;68:305-315.

Publisher's Note Springer Nature remains neutral with regard to jurisdictional claims in published maps and institutional affiliations. 